\title{
Injector for highly viscous silicone oil
}

\author{
Norbert Hausmann, Gisbert Richard
}

\begin{abstract}
A syringe device for the injection and evacuation of highly viscous silicone oil was developed. The problem of removing the air interfering with these manoeuvres was solved by providing special outlets in the plunger and handle of a conventional glass syringe. A slightly modified commercial motor perfusor was chosen to power this device, which has proved to be good and fail-safe in more than 100 cases.
\end{abstract}

The main types of silicone oil injected in complicated proliferative vitreoretinopathy cases after vitrectomy now have viscosities of 1000 and 5000 (highly purified) centistokes. ${ }^{1}$ Owing to the high viscosity, an injection syringe can be filled properly only with its face down from behind after removal of the plunger; simple aspiration will not work. To evacuate the air, then located between oil level and plunger after reassembling the syringe, part of the silicone oil must be squeezed out - the syringe now faced upwards - together with the air locked in the entire system. This necessitates a considerable sacrifice of time and loss of oil. Further, owing to the high viscosity of the 5000 centistokes oil in particular, a strong and reliable motor which works in both directions is required, and which, besides injection, also ensures evacuation of oil in the same way. In our view there is no instrument on the market that meets these requirements at a reasonable price. We have therefore designed the instrument described below.

Figure 1 Top: Inverse cone and borehole ground into the plunger, external thread and lateral outlet on handle (arrows), unscrewed concentric tube. Bottom: Tube screwed down along the thread past the lateral outlet. between oil level and plunger we took a conventional glass syringe and bored through the centre of its plunger and handle. The cone of the plunger was changed, so that its top pointed then to the bore hole, where the air could now accumulate. Above the plunger a right-angled outlet was added laterally in the first third of the handle leading the bore out. To make this outlet tight we have slipped a movable concentric tube with an internal thread over the handle, which in its turn was provided with a corresponding external thread (Fig 1). The syringe is now filled from behind in the usual way when the plunger is removed. The plunger is then inserted with the concentric tube on its handle unscrewed and finally slid down to the oil level. In this way the air above the oil can accumulate in the plunger's cone and escape through the lateral outlet. When the syringe is void of air, the concentric tube is screwed down along the thread past the outlet. This makes the system airtight (Fig 1). Through a pressure-proof Teflon hose the silicone oil passes into the vitrectomised eye, either directly via the infusion line or through a blunt needle controlled by the surgeon's hand. The technique of fluid exchange has been described elsewhere. ${ }^{2}$

A syringe for silicone oil injection should no longer be manually operated by screwing a spindle, as earlier publications on this topic recommend..$^{3-5}$ Commercial quality power sources are either battery operated and therefore susceptible to trouble or have a rather expensive mains connection. ${ }^{6}$ For these reasons we have chosen a time tested motor perfusor, which is at hand in every hospital for the precise control of thrombolysis by streptokinase to power our syringe. It was fastened to the perfusor by a cross bar at the tip and by a cone and a screw at the handle (Fig 2). The power of the perfusor will allow a choice among several speeds of injection, even for the highly viscous 5000 centistokes oil. The same applies, for example, to evacuation in a case of oil bubble behind the retina. Owing to the high suction power needed for this manoeuvre an increasing concentration of air in the syringe is unavoidable, even if the plunger has been ground in with the utmost precision. Therefore the plunger soon comes to a stop, leaving a mostly air filled and functionally disturbed syringe.

With our device removal of air during oil evacuation is as simple as is oil filling: The whole instrument is tipped down by a surgical nurse to accumulate the air again between the oil level and the plunger. The concentric tube on the handle is then unscrewed and the plunger slid forward again by the activated motor. Thus the air is squeezed out through the handle's outlet. As soon as the air has escaped and the tube is tightened, the evacuation can continue with a normally positioned instrument. During this manoeuvre the surgeon can keep the evacuation 


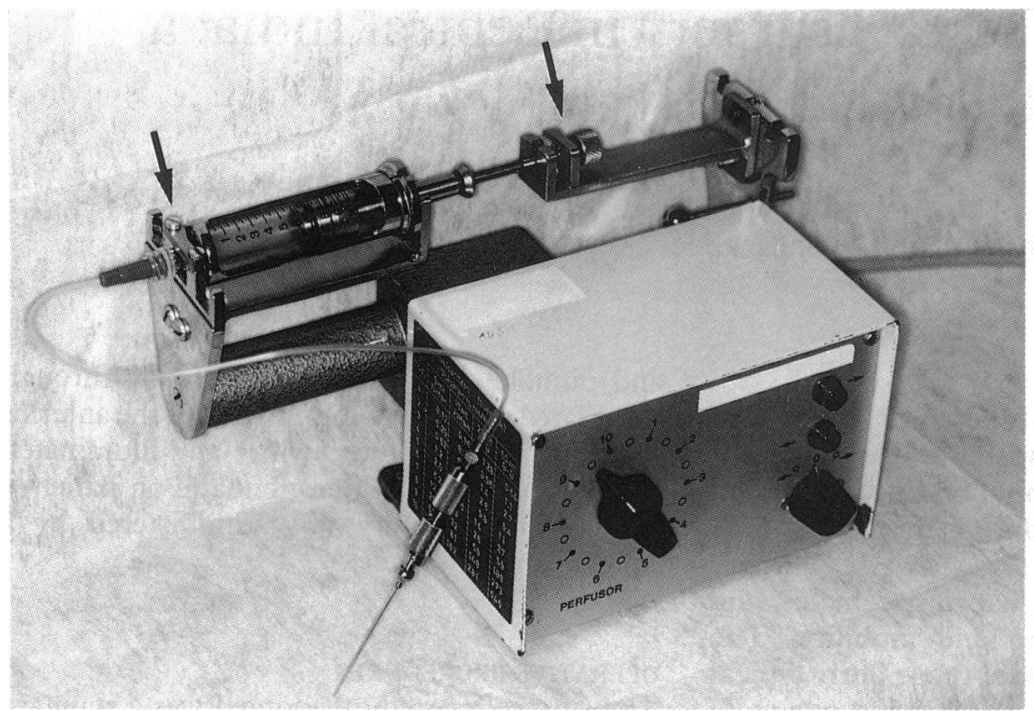

Figure 2 The oil filled, airless syringe fastened to the motor perfusor by a cross bar at the tip and by a cone and $a$ screw at the handle (arrows). needle in the eye. Thus he need not squeeze the air back through the complete system and the withdrawn needle.

The device described here is not commercially produced. It can, however, be made at a slight cost from supplies available at every hospital by any skilful mechanic. So far it has proved to be good and safe in more than 100 cases.

The authors have no proprietary or financial interest in the instrument. We acknowledge the constructional assistance and mechanical work done by our male nurse, Mr Djuro Bajic, at Feldkirch, Austria.

1 Gabel VP, Kampik A, Gabel C. Silicone oil with high specific gravity for intraocular use. BrF Ophthalmol 1987; 71: 143-8. 2 Charles S. Fluid-gas exchange in the vitreous cavity. Ocutome Newsletter 1977; 2: 1-3.

3 Cibis PA, Becker B, Okun E, Canaan S. The use of liquid silicone in retinal detachment surgery. Arch Ophthalmol 1962; 68: $590-9$

4 Kelly TSB. Screw type syringe for intravitreous injection. Mod Probl Ophthalmol 1969; 8: 577-80.

Scott JD. Treatment of detached immobile retina Trans Ophthalmol Soc UK 1972; 92: 351-7.

6 Zivojnovic R. Silicone oil in vitreoretinal surgery. Lancaster: Kluwer Academic, 1987: 25-7. 